\title{
UN ENFOQUE BAYESIANO PARA INCORPORAR PRONÓSTICOS DE LA DEMANDA EN EXPERIMENTOS POR SIMULACIÓN PARA LA ADMINISTRACIÓN DE INVENTARIOS
}

\author{
David Muñoz N. ${ }^{1}$ \\ Recibido el 6 de enero de 2004, aceptado el 2 de marzo de 2004
}

\begin{abstract}
RESUMEN
Con el objetivo de planear la producción y distribución de manufacturas con base en información cada vez más cercana al momento de venta, los sistemas de apoyo a la toma de decisiones para la administración de inventarios deben incorporar pronósticos de la demanda basados en poca información objetiva, o en información subjetiva. En particular, cuando se utilizan modelos de simulación para apoyar toma de decisiones relacionadas con inventarios de seguridad, o con tamaños y demoras de los pedidos, es conveniente modelar la distribución de la demanda tomando en cuenta tanto los datos disponibles sobre experiencias pasadas, como la información (a menudo subjetiva) sobre el futuro cercano. En este artículo se presenta un enfoque bayesiano para modelar un componente aleatorio de entrada (por ejemplo, la distribución de la demanda) en experimentos por simulación para la administración de inventarios. Bajo este enfoque, la familia de distribuciones propuesta para modelar el componente de entrada debe considerar dos tipos de parámetros, los que capturan información de datos históricos y los que dependen del pronóstico (a menudo subjetivo) sobre el escenario particular a simular. La aplicación del enfoque propuesto se ilustra con un ejemplo en el que se modela la demanda diaria por medio de una distribución binomial negativa, y el usuario del sistema proporciona la demanda esperada para todo el periodo a simular .
\end{abstract}

Palabras claves: Simulación de inventarios, pronóstico de demanda, estimación bayesiana, cadena de suministro.

\begin{abstract}
In order to postpone production planning based on information obtained closer to the time of sale, decision support systems for inventory management often include demand forecasts based on little historical data and/or subjective information. Particularly, when simulation models for analyzing decisions related to safety inventories, lot sizing or lead times are used, it is convenient to model demand considering historical data, as well as information (often subjective) of the near future. This article presents an approach for modeling a random input component (e.g., demand) in simulation experiments for inventory management. Under this approach, the family of distributions proposed for modeling the random component include two types of parameters: the ones that capture information of historical data and the ones that depend on forecasts (often subjective) from the particular scenario that is to be simulated. The application of the proposed approach is illustrated with an example which models daily demand through a negative binomial distribution, where the system user provides the expected demand for the period that is to be simulated.
\end{abstract}

Keywords: Inventory simulation, demand forecast, bayesian estimation, supply chain.

\section{INTRODUCCIÓN}

Cuando se evalúa el desempeño de una política de administración de inventarios a través de un modelo, la incorporación de algunas características del sistema real puede dificultar la obtención de soluciones analíticas para las medidas de desempeño. Por ejemplo, cuando se investiga la relación entre inventarios de seguridad, demoras de los pedidos y niveles de servicio en diferentes puntos de la cadena de suministro, a menudo los patrones de demanda cambian en el tiempo, los pedidos no son siempre del mismo tamaño (dependen del pronóstico de ventas), o existe incertidumbre sobre la demora en la atención de los pedidos debido al tamaño de los mismos, o a restricciones propias de las unidades de producción. Estas características, sin embargo, a menudo pueden incorporarse en un modelo de simulación, con la finalidad de estudiar el desempeño

\footnotetext{
${ }^{1}$ Departamento de Ingeniería Industrial y de Operaciones, Instituto Tecnológico Autónomo de México, México D.F., México, davidm@itam.mx
} 
de la política de inventarios a través de la experimentación con el modelo de simulación, y debido a su capacidad para modelar sistemas complejos, la simulación es una herramienta eficaz para evaluar el desempeño de una política de administración de inventarios (véase, por ejemplo, [3]).

Para conducir un experimento por simulación, deben especificarse de manera precisa tanto los componentes de entrada como las salidas (medidas de desempeño) del sistema a estudiar. En particular, si se desea simular los inventarios en una cadena de suministro (Fig. 1), a menudo las medidas de desempeño (salidas) del experimento están asociadas con los niveles de servicio y con los costos incurridos, y para estimar estas medidas de desempeño se deben simular los niveles de inventario con base en la política de inventarios (en particular, de inventarios de seguridad), y en las distribuciones de probabilidad tanto de la demora de los pedidos como de la demanda (ya sea de los clientes individuales o por periodo, por ejemplo, diaria). Es conveniente remarcar que algunos componentes de entrada del modelo pueden ser conocidos (el nivel de inventario inicial, el sistema de revisión, etc.), y otros podrían estar definidos por una distribución de probabilidades, estos últimos a menudo son llamados componentes aleatorios de entrada del modelo [9].

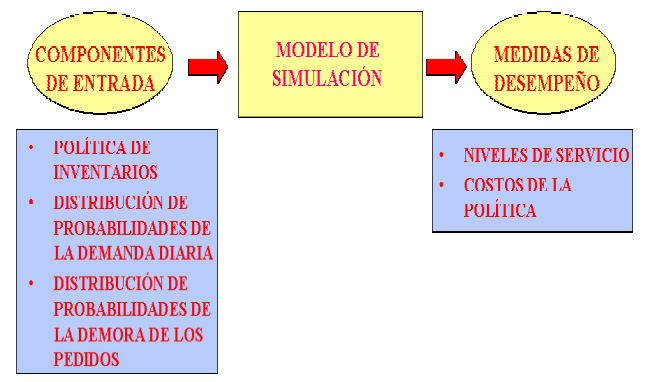

Fig. 1.- Elementos de un experimento por simulación para analizar inventarios

El enfoque clásico para especificar la distribución de un componente aleatorio de entrada en un experimento por simulación consiste en seleccionar la distribución g (y sus parámetros) que se "ajusta" mejor a un conjunto de observaciones del componente, las que a menudo se asumen independientes e idénticamente distribuidas (i.i.d.). Para ser más precisos, a menudo se propone el ajuste de los parámetros con base en el método de máxima verosimilitud, y se escoge la distribución de probabilidades que se comporte mejor de acuerdo a alguna medida de bondad de ajuste como el error cuadrático promedio (véase, por ejemplo, [7]).

Sin embargo, cuando se desea modelar la demanda de productos con ciclo de vida cortos (productos de moda o de temporada), o de productos cuya demanda depende de factores del entorno (dinero en agencias bancarias u otros productos con cartera de clientes), no es conveniente asumir que las observaciones previas de la demanda provienen de la misma distribución, ya que es más realista asumir que los parámetros de la distribución de cada subconjunto de observaciones dependen de los factores propios del entorno en el periodo al que pertenecen las observaciones. En este caso, el enfoque clásico resulta inapropiado para modelar la demanda del producto, y debe buscarse una metodología que permita incorporar las características propias de la distribución de la demanda correspondiente al escenario que se desea simular. Cabe mencionar que algunas empresas (en particular, del sector de confecciones de moda) tienen una estrategia agresiva de ventas basada en su capacidad para manejar sus pronósticos de ventas, sus inventarios, y una cadena de suministro veloz [5].

En este artículo se presenta una metodología (con fundamento en la Estadística Bayesiana) para modelar un componente aleatorio de entrada (en particular la demanda) en experimentos por simulación. Bajo este enfoque, la distribución del componente a modelar depende de dos tipos de parámetros, los primeros proporcionan información que es común a las observaciones previas del componente aleatorio, y los segundos proporcionan información particular del escenario a simular. A continuación, se muestra cómo, con base en el modelo propuesto, información histórica disponible y probabilidades a priori sobre los parámetros del modelo, se pueden combinar para obtener probabilidades a posteriori para los parámetros del modelo, las que permiten estimar las medidas de desempeño del experimento por simulación.

Luego de la introducción, en la segunda sección se describe la notación a utilizar y la metodología propuesta; en la tercera sección se presenta un ejemplo basado en el caso que ha motivado el desarrollo de la metodología propuesta, con el propósito de ilustrar la aplicación del presente enfoque, y finalmente en la cuarta sección se presentan las conclusiones, y se discute la investigación necesaria para sustentar la aplicación práctica de este enfoque.

\section{EXPERIMENTOS POR SIMULACIÓN BAJO UN ENFOQUE BAYESIANO}

La metodología que a continuación se describe tiene por finalidad determinar cómo se podría especificar la distribución de un componente aleatorio de entrada (por ejemplo, la demanda diaria), tratando de incorporar tanto información particular del escenario de interés 
(pronóstico de la demanda total en el periodo a simular) como información histórica (registros disponibles de las demandas diarias). Con este propósito, se asume que se dispone de $Q$ muestras (independientes entre sí), $x_{i}=\left(x_{i 1}, x_{i 2}, x_{i n_{i}}\right)$, para $i=1,2, \ldots, Q$, de observaciones del componente aleatorio de entrada (demanda diaria) correspondientes a $Q$ periodos (meses) anteriores, respectivamente, donde las observaciones dentro de la muestra $i$ son i.i.d., y provenientes de la función de probabilidades $p\left(y ; \Theta_{1}, \Theta_{2}^{i}\right)$ (Fig. 2), siendo que el valor del parámetro $\Theta_{1}$ es el mismo para todas las muestras (inclusive para las que se van a generar por simulación), y el valor del parámetro $\Theta_{2}^{i}$ corresponde al escenario particular del $i$-ésimo periodo (mes). Es decir, la función de probabilidades (densidad en el caso continuo) que se propone para el componente aleatorio de entrada (demanda diaria) tiene la forma $p\left(y ; \Theta_{1}, \Theta_{2}\right)$, donde $\Theta_{1}$ (de dimensión $\left.k_{1}\right)$ es el vector de parámetros que sirven para modelar la información de experiencias pasadas, y $\Theta_{2}$ (de dimensión $k_{2}$ ) es el vector de parámetros que modelan la información del escenario particular que se desea simular. Es conveniente indicar que, de acuerdo con la Fig. 2, $p\left(y ; \Theta_{1}, \Theta_{2}^{Q+1}\right)$ identifica a la función de probabilidades del componente aleatorio de entrada correspondiente al escenario a simular.



Fig. 2.- Estructura de la información histórica disponible

Por otro lado, la información (pronóstico) a priori sobre los parámetros se expresa por medio de una función de densidad a priori $p_{\Theta}\left(\theta_{1}, \theta_{2}^{1}, \theta_{2}^{2}, \ldots \theta_{2}^{Q+1}\right)$, que (asumiendo independencia) tiene la forma:

$$
\begin{aligned}
\mathrm{p}_{\Theta}(\theta) & =\mathrm{p}_{\Theta}\left(\theta_{1}, \theta_{2}^{1}, \theta_{2}^{2}, \ldots \theta_{2}^{\mathrm{Q}+1}\right) \\
& =\mathrm{p}_{\Theta_{1}}\left(\theta_{1}\right) \mathrm{p}_{\Theta_{2}^{1}}\left(\theta_{2}^{1}\right) \mathrm{p}_{\Theta_{2}^{2}}\left(\theta_{2}^{2}\right) \ldots \mathrm{p}_{\Theta_{2}^{\mathrm{Q}+1}}\left(\theta_{2}^{\mathrm{Q}+1}\right)
\end{aligned}
$$

donde $\theta=\left(\theta_{1}, \theta_{2}^{1}, \ldots, \theta_{2}^{Q+1}\right), y \quad \Theta=\left(\Theta_{1}, \Theta_{2}^{1}, \ldots, \Theta_{2}^{Q+1}\right)$.
Es conveniente mencionar que el pronóstico sobre el parámetro de escenario $\Theta_{2}^{i}$ correspondiente al periodo $i$, a menudo se puede expresar a través de una función de densidad $p_{\Theta_{2}^{i}}\left(\theta_{2}^{i}\right), i=1,2, \ldots, Q$, ya que existe una gran variedad de maneras en las que se puede identificar un pronóstico sobre un parámetro por medio de la especificación de una función de densidad a priori. Por ejemplo, se puede asumir una distribución que toma cierto valor con probabilidad 1 (apropiada para el caso en que el parámetro se conoce con certeza), o una distribución uniforme en cierto intervalo (cuando sólo se pueden pronosticar los valores mínimo y máximo del parámetro), o cualquier otra forma que se adapte mejor al método de pronóstico utilizado.

Utilizando la notación previa, podemos indicar que, una salida $y$ (por ejemplo, el porcentaje de la demanda atendida) de un experimento por simulación, puede concebirse como una función de los números aleatorios $u$ utilizados en la corrida, y del valor particular $\theta^{Q+1}=\left(\theta_{1}, \theta_{2}^{Q+1}\right)$ de los parámetros del componente aleatorio de entrada:

$$
y=y\left(u, \theta^{Q+1}\right)
$$

Como se discute en [9], bajo un enfoque clásico (no bayesiano), a menudo se fija el valor $\theta^{Q+1}$ de los parámetros, y se corren repeticiones independientes del experimento por simulación, para estimar medidas de desempeño de la forma:

$$
\eta\left(\theta^{Q+1}\right)=E\left[y \mid \Theta^{Q+1}=\theta^{Q+1}\right]=\int y\left(u, \theta^{Q+1}\right) d u
$$

En cambio, bajo un enfoque bayesiano, los parámetros son variables aleatorias, y se estiman medidas de desempeño de la forma:

$$
E[y \mid X=x]=\int \eta\left(\theta^{Q+1}\right) p_{\Theta^{Q+1} \mid X=x}\left(\theta^{Q+1}\right) d \theta^{Q+1}
$$

donde $x=\left(x_{1}, x_{2}, \ldots, x_{Q}\right)$ denota al vector de observaciones disponibles (información histórica), y $p_{\Theta^{Q+1} \mid X=x}\left(\theta^{Q+1}\right)$ denota la función de densidad a posteriori (dada la información histórica) de los parámetros del escenario a simular, la cual se puede obtener a partir de la función de densidad a priori de los parámetros, y de la información histórica $x$, como se detalla a continuación.

Como las observaciones $x_{i}=\left(x_{i 1}, x_{i 2}, \ldots, x_{i n_{i}}\right)$ dentro de cada muestra $i=1,2, \ldots, Q$ son i.i.d., y las $Q$ muestras 
son independientes entre si, dado que el conjunto de parámetros $\Theta$ toma el valor $\theta=\left(\theta_{1}, \theta_{2}^{1}, \ldots, \theta_{2}^{Q+1}\right)$, la función de probabilidades conjunta de la información disponible $x$ es:

$$
p_{X \mid \Theta=\theta}(x)=\prod_{i=1}^{Q} \prod_{j=1}^{n_{i}} p\left(x_{i j} ; \theta_{1}, \theta_{2}^{i}\right)
$$

por lo que

$$
\begin{aligned}
p_{x}(x)= & \int \prod_{i=1}^{Q} \prod_{j=1}^{n_{i}} p\left(x_{i j} ; \theta_{1}, \theta_{2}^{i}\right) \\
& p_{\Theta}\left(\theta_{1}, \theta_{2}^{l}, \ldots \theta_{2}^{Q+1}\right) d \theta_{1} d \theta_{2}^{l} \ldots d \theta_{2}^{Q+1}
\end{aligned}
$$

donde la función de densidad a priori $p_{\Theta}\left(\theta_{1}, \theta_{2}^{1}, \ldots, \theta_{2}^{Q+1}\right)$ se define en la ecuación (1).

De acuerdo con el Teorema de Bayes [1], la función de densidad a posteriori conjunta de los parámetros resulta:

$$
p_{\Theta \mid X=x}(\theta)=\frac{p_{\Theta}(\theta) p_{X \mid \Theta=\theta}(x)}{p_{X}(x)}
$$

donde $p_{\Theta}(\theta), \quad p_{X \mid \Theta=\theta}(x)$, y $p_{X}(x)$ están definidas en las ecuaciones (1), (5) y (6), respectivamente. Por último, la función de densidad a posteriori de los parámetros $\theta^{Q+1}=\left(\theta_{1}, \theta_{2}^{Q+1}\right)$ del escenario a simular se obtienen de:

$$
p_{\Theta^{Q+1} \mid X=x}\left(\theta^{Q+1}\right)=\int p_{\Theta \mid X=x}(\theta) d \theta_{2}^{1} \ldots d \theta_{2}^{Q}
$$

donde $p_{\Theta \mid X=x}(\theta)$ se define en (7).

Nótese ahora que el parámetro $E[y \mid X=x]$ (que se desea estimar) definido en la ecuación (4) es una esperanza, por lo que se puede estimar por simulación haciendo uso del método de repeticiones, es decir, se obtienen $n$ repeticiones independientes del siguiente experimento:

- Generar un vector de parámetros $\theta^{Q+1}=\left(\theta_{1}, \theta_{2}^{Q+1}\right)$ de acuerdo con la función de densidad a posteriori $p_{\Theta^{Q+1} \mid X=x}\left(\theta^{Q+1}\right)$ definida en (8).
- Correr el modelo de simulación y generar la salida $y=y\left(u, \theta^{Q+1}\right)$.

Un estimador consistente para la medida de desempeño $E[y \mid X=x]$ es el promedio simple de las $n$ repeticiones independientes del experimento anterior [2]. Es conveniente mencionar que la metodología descrita en esta sección está inspirada en la metodología desarrollada en [2] para seleccionar la distribución de un componente aleatorio de entrada en experimentos por simulación, aplicando la técnica denominada Bayesian Model Average [4], con la diferencia que en [2] se proponen diferentes modelos para el mismo componente aleatorio, cada modelo tiene un solo tipo de parámetro, y se dispone de una sola muestra para obtener la distribución a posteriori. Mientras que en [2] el énfasis radica en incorporar la incertidumbre sobre el modelo apropiado para el componente aleatorio de entrada, el caso descrito en este artículo pone énfasis más bien en la forma en que se puede incorporar información de pronóstico (a lo mejor subjetiva) sobre un escenario de interés, asumiendo la existencia de varias $(Q)$ muestras (bajo diferentes escenarios). Por otro lado, cabe mencionar que la metodología propuesta en este artículo se puede extender naturalmente para el caso en que se deseen proponer diferentes modelos para el mismo componente aleatorio de entrada, aplicando la técnica BMA [8], en cuyo caso se pueden proponer también algoritmos alternativos para la estimación de la medida de desempeño de interés, e incluso analizar las fuentes de variabilidad del experimento por simulación [9].

Es conveniente indicar que si bien la única diferencia (con el método clásico de repeticiones) que induce el enfoque bayesiano en el experimento por simulación, es que ahora el parámetro debe muestrearse de la distribución a posteriori (8) antes de ejecutar cada corrida, como se discute, por ejemplo en [2] y [9], el denominador en (7) pudiera no tener una expresión analítica sencilla, y debería evaluarse haciendo uso de métodos numéricos. Cuando éste es el caso, diversos autores (por ejemplo, [2] y [9]) recomiendan el uso de la técnica conocida por las siglas MCMC (del término inglés Markov Chain Monte Carlo), que en lugar de generar muestras independientes de la distribución a posteriori (8), consiste básicamente en generar muestras de una cadena de Markov que tiene distribución estacionaria igual a (8), y que es más fácil de generar que de (8) directamente, de manera que el estimador que se obtiene es también consistente, de acuerdo con la Ley de los Grandes Números para Cadenas de Markov [6]. Otro método alternativo (y fácil de aplicar) cuando el denominador de (7) tiene una expresión complicada, se basa en el uso de la técnica llamada muestreo de importancia (del término inglés importance sampling). 
Como se explica en [1], este método se puede aplicar si se propone una función de densidad $h_{\Theta}(\theta)$ de la cual es fácil generar una muestra del vector de parámetros $\Theta$, observando que para cualquier función (medible) $g(\theta)$ se cumple que:

$$
\begin{aligned}
& E_{h}\left[\frac{g(\theta) p_{\Theta}(\theta) p_{x \mid \Theta=\theta}(\mathrm{x})}{h_{\Theta}(\theta)}\right]= \\
& =\int \mathrm{g}(\theta) \mathrm{p}_{\Theta}(\theta) \mathrm{p}_{\mathrm{x} \mid \Theta=\theta}(\mathrm{x}) \mathrm{d} \theta_{1} \mathrm{~d} \theta_{2}^{1} \ldots \mathrm{d} \theta_{2}^{\mathrm{Q}+1}
\end{aligned}
$$

de acuerdo con la Ley de los Grandes Números, un estimador consistente para el lado izquierdo de (9) es el promedio (luego de generar una muestra $\left\{u_{1}, \ldots, u_{n}\right\}$ de $n$ observaciones de $\Theta$ a partir de la densidad $h_{\Theta}(\theta)$ ):

$$
\frac{1}{n} \sum_{i=!}^{n} \frac{g\left(u_{i}\right) p_{\Theta}\left(u_{i}\right) p_{X \mid \Theta=u_{i}}(x)}{h_{\Theta}\left(u_{i}\right)}
$$

Por otro lado, a partir de (4) y (8) se puede ver que:

$$
E[y \mid X=x]=\frac{\int \eta\left(\theta^{Q+1}\right) p_{\Theta}(\theta) p_{x \mid \Theta=\theta}(x) d \theta_{1} d \theta_{2}^{1} \ldots d \theta_{2}^{Q+1}}{\int p_{\Theta}(\theta) p_{X \mid \Theta=\theta}(x) d \theta_{1} d \theta_{2}^{1} \ldots d \theta_{2}^{Q+1}}
$$

por lo que $E[y \mid X=x]$ se puede estimar aproximando el numerador y el denominador de (11), tomando $g(\theta)=\eta\left(\theta^{Q+1}\right)$, y $g(\theta)=1$ en $(10)$, respectivamente.

\section{UN EJEMPLO}

La motivación para desarrollar la presente metodología surge del caso de una empresa de la industria de manufacturas que produce cierto(s) artículo(s) (con diseño estandarizado) bajo pedido, para una cartera de clientes. Al inicio de cada mes la empresa produce estimados de la demanda mensual con base en la información proporcionada por sus clientes, siendo que cierta información puede corresponder a pedidos adelantados, aunque sin identificar la fecha exacta ni los tamaños de las entregas, mismos que serán especificados durante el transcurso del mes. Con base en los estimados iniciales de la demanda agregada del mes, y en su política de inventarios de seguridad, la empresa establece su plan de producción para satisfacer los pedidos durante el mes, por lo que la empresa está interesada en desarrollar un modelo de simulación que le permita estimar el nivel de servicio (en este caso, el porcentaje esperado de demanda satisfecha) para una política de producción establecida, y un escenario de demanda congruente con los pronósticos agregados del mes y con la información del comportamiento de la demanda en meses pasados.

La empresa dispone de información del pronóstico (agregado mensual) y de la demanda real (diaria) para $Q$ meses anteriores; cada mes se ha registrado la siguiente información:

$\mathrm{S}_{\mathrm{i}}$ : pronóstico de la demanda total en el mes $i$, $\mathrm{X}_{\mathrm{ij}}$ : demanda (en unidades) en el día $j$ del mes $i$, $j=1,2, \ldots, n_{i}, \quad i=1,2, \ldots, Q$. Se propone que la distribución de la demanda diaria en un mes determinado sigue una distribución binomial negativa:

$$
p\left(y, \Theta_{1}, \Theta_{2}\right)=\frac{\overline{\left(\Theta_{2}+y\right)}}{\sqrt{(1+y)}\left(\Theta_{2}\right)} \Theta_{1}^{\theta_{2}}\left(1-\Theta_{1}\right)^{y}, y=0,1, \ldots,
$$

donde $0<\Theta_{1}<1$ es un parámetro de forma (común en todos los meses), $\Theta_{2}>0$ es un parámetro de escala que depende del pronóstico (agregado) del mes, y $\longdiv { ( x ) }$ denota a la función gamma evaluada en $x>0$. Por otro lado, para que el pronóstico del mes $i$ sea congruente con el parámetro de escala, suponemos que dado que el pronóstico del mes $i$ es $S_{i}$, el parámetro $\Theta_{2}^{i}$ toma el valor $\frac{S_{i} \Theta_{1}}{n_{i}\left(1-\Theta_{1}\right)}$ con probabilidad 1 (que equivale a establecer que la demanda esperada en cualquier día del mes $i$ es $S_{i} / n_{i}$ ).

Se ha desarrollado un modelo de simulación para la empresa que permite estimar el nivel de servicio (porcentaje de demanda satisfecha) $y=y\left(u, \theta^{Q+1}\right)$ (donde $\theta^{Q+1}=\left(\theta_{1}, \theta_{2}^{Q+1}\right)$ ), cuando se utiliza la política actual de administración de inventarios. Dado que para el próximo mes se pronostica una demanda total de $S_{Q+1}$ unidades, se desea utilizar el modelo para estimar el nivel de servicio $E[y \mid X=x]$ dada la información de las experiencias anteriores (en particular, se sabe que $\left.\Theta_{2}^{i}=\frac{S_{i} \Theta_{1}}{n_{i}\left(1-\Theta_{1}\right)}, i=1,2, \ldots Q\right)$.

Nótese que la información sobre el parámetro de forma $\Theta_{1}$ está presente en todos los meses anteriores, mientras que el parámetro de escala $\Theta_{2}^{i}, \quad i=1,2, \ldots Q+1$, es información particular de cada escenario. 
Como se tiene registro del pronóstico de la demanda total en cada mes, la función de densidad a priori apropiada para los parámetros $\Theta=\left(\Theta_{1}, \Theta_{2}^{1} \ldots, \Theta_{2}^{Q+1}\right)$, está dada por:

$$
\mathrm{p}_{\Theta}\left(\theta_{1}, \theta_{2}^{1}, \ldots \theta_{2}^{\mathrm{Q}+1}\right)=1
$$

donde

$$
0<\theta_{1}<1, \theta_{2}^{1}=\frac{\mathrm{S}_{1} \theta_{1}}{\mathrm{n}_{1}\left(1-\theta_{1}\right)}, \ldots, \theta_{2}^{\mathrm{Q}+1}=\frac{\mathrm{S}_{\mathrm{Q}+1} \theta_{1}}{\mathrm{n}_{\mathrm{Q}+1}\left(1-\theta_{1}\right)}
$$

misma que no proporciona información sobre el parámetro de forma $\Theta_{1}$, pero incorpora la información sobre el pronóstico (agregado) de la demanda mensual.

Teniendo en cuenta las suposiciones (12) y (13), y de acuerdo con las ecuaciones (5) y (9), la función de probabilidades conjunta de la información disponible dado el valor de los parámetros es:

$$
p_{X \mid \Theta=\theta}(x)=\prod_{i=1}^{Q} \prod_{j=1}^{n_{i}} \frac{\sqrt{\left(\theta_{2}^{i}+x_{i j}\right)}}{\left(1+x_{i j}\right)\left(\theta_{2}^{i}\right)} \theta_{1}^{\theta_{2}^{i}}\left(1-\theta_{1}\right)^{x_{i j}} \text {, }
$$

y de acuerdo con las ecuaciones (6) y (10), se obtiene:

$$
p_{X}(x)=\int_{0}^{1} \prod_{i=1}^{O} \prod_{j=1}^{n} \frac{\sqrt{\left(u_{i}\left(\theta_{1}\right)+x_{i j}\right)}}{\sqrt{\left.\left(1+x_{i j}\right)\right)\left(u_{i}\left(\theta_{1}\right)\right)}} \theta_{1}^{u_{i}\left(\theta_{1}\right)}\left(1-\theta_{1}\right)^{x_{i}} d \theta_{1}
$$

donde $u_{i}\left(\theta_{1}\right)=\frac{S_{i} \theta_{1}}{n_{i}\left(1-\theta_{1}\right)}, i=1,2, \ldots Q$. Teniendo ahora en cuenta (7), (10), (13) y (14) se obtiene:

$$
\begin{aligned}
& \mathrm{p}_{\Theta^{Q+1} \mid \mathrm{X}=\mathrm{x}}\left(\theta_{1}, \theta_{2}^{\mathrm{Q}+1}\right)= \\
& =\frac{\prod_{\mathrm{i}=1}^{\mathrm{Q}} \prod_{\mathrm{j}=1}^{\mathrm{n}_{\mathrm{i}}} \frac{\sqrt{\left(\mathrm{u}_{\mathrm{i}}\left(\theta_{1}\right)+\mathrm{x}_{\mathrm{ij}}\right)}}{\sqrt{\left(1+\mathrm{x}_{\mathrm{ij}}\right)} \sqrt{\left(\mathrm{u}_{\mathrm{i}}\left(\theta_{1}\right)\right)}} \theta_{1}^{\mathrm{u}_{\mathrm{i}}\left(\theta_{1}\right)}\left(1-\theta_{1}\right)^{\mathrm{x}_{\mathrm{ij}}}}{\mathrm{p}_{\mathrm{X}}(\mathrm{x})} \\
& \text { para } 0<\theta_{1}<1, \theta_{2}^{Q+1}=\frac{S_{Q+1} \theta_{1}}{n_{Q+1}\left(1-\theta_{1}\right)}, \text { y } p_{\mathrm{X}}(x) \text { definido en }
\end{aligned}
$$
(13).

De acuerdo con la metodología descrita en la sección anterior, el costo esperado $E[y \mid X=x]$ dada la información de las experiencias anteriores puede estimarse por simulación, repitiendo el siguiente experimento:

- Generar un vector de parámetros $\theta^{Q+1}=\left(\theta_{1}, \theta_{2}^{Q+1}\right)$ de acuerdo con la distribución a posteriori $p_{\Theta^{Q+1} \mid X=x}\left(\theta^{Q+1}\right)$ definida en (15).

- Correr el modelo de simulación y generar una observación del costo $y=y\left(u, \theta^{Q+1}\right)$.

El estimador buscado es el promedio de las observaciones del costo $y=y\left(u, \theta^{Q+1}\right)$ generadas por simulación.

Nótese que el denominador de (15) es complicado de evaluar, aunque si se aplica muestreo de importancia aprovechando que $0<\theta_{1}<1$, se puede probar una distribución uniforme entre 0 y 1 , es decir, $h_{\Theta_{1}}\left(\theta_{1}\right)=1,0<\theta_{1}<1$, de manera que el método descrito al final de la sección anterior, puede resultar relativamente sencillo de implantar, para evaluar el nivel de servicio $E[y \mid X=x]$, dada la información histórica disponible y el pronóstico de demanda para el próximo mes.

\section{CONCLUSIONES E INVESTIGACIÓN A FUTURO}

Como se describe en la segunda sección, un enfoque bayesiano permite incorporar tanto información correspondiente a un escenario de interés (a través de un vector de parámetros $\theta_{2}^{Q+1}$ y su correspondiente distribución a priori), como información sobre experiencias pasadas (a través de un parámetro común $\theta_{1}$ y su correspondiente distribución a priori). Dado un modelo propuesto para un componente aleatorio de entrada, la diferencia fundamental entre un enfoque clásico y el enfoque bayesiano propuesto radica en que mientras en el enfoque clásico se fijan los valores de los parámetros del modelo, en el enfoque bayesiano los parámetros se muestrean de acuerdo con una distribución a posteriori, y la pregunta fundamental a responder es por qué podría ser conveniente este nuevo enfoque. Una respuesta intuitiva puede ser que si existe incertidumbre en el valor de los parámetros, el enfoque clásico debe tender a subestimar la variabilidad del estimador hallado. Sin embargo, una respuesta científica a esta pregunta debe sustentarse en evidencia sólida, por lo que se requiere de una investigación más detallada (a 
lo mejor empírica) para dar respuesta a esta pregunta; al respecto se sugiere consultar [2].

La principal motivación para desarrollar el marco teórico presentado en la segunda sección, ha sido la de incorporar tanto información de pronóstico del usuario de un modelo de simulación, como evidencia empírica para tomar decisiones sobre un escenario propuesto. Sin embargo, la utilidad del sistema de apoyo a la toma de decisiones estará íntimamente ligada a su capacidad para modelar el sistema real, la cual dependerá de una buena elección de los parámetros de entorno $\theta_{2}^{i}$, de la elección de la información que proporcionan los datos históricos (reflejada en el parámetro común $\theta_{1}$ ), y de una buena elección del modelo $p\left(y, \Theta_{1}, \Theta_{2}\right)$, las que indudablemente dependen del problema particular a resolver. Es justamente en esta área, la búsqueda de modelos y aplicaciones particulares de los mismos, en la que se encuentra el mayor potencial de investigación relacionada con la metodología propuesta.

\section{AGRADECIMIENTOS}

Esta investigación ha sido patrocinada por la Asociación Mexicana de Cultura A.C.

\section{REFERENCIAS}

[1] J.O. Berger; "Statistical Decision Theory and Bayesian Analysis", Springer-Verlag, New York, 2a. Edición, 1985.

[2] S.E. Chick; "Input distribution selection for simulation experiments: accounting for input uncertainty", Operations Research, Vol. 49, No. 5, pp. 744-758, 2001.

[3] S. Chopra and P. Meindl; "Supply Chain Management", Prentice-Hall Inc., New Jersey, 2001 .

[4] D. Draper; "Assessment and propagation of model uncertainty (with discussion)", J. of the Royal Statist. Soc., Series B, Vol. 57, No. 1, pp. 45-97, 1995.

[5] M. L. Fisher, A. Raman and A.S. McClelland; "Rocket science retailing is almost here", Harvard Business Review, July-August, pp. 115-124, 2000.

[6] W.R. Gilks, S. Richardson and D.J. Spiegelhalter; "Markov Chain Monte Carlo in Practice", Chapman and Hall, Londres, 1996.
[7] A.M. Law and W.D. Kelton; "Simulation Modeling and Analysis", McGraw-Hill, New York, 3a. Edición, 2000.

[8] D.F. Muñoz; "A Bayesian framework for modeling demand in supply chain simulation experiments", S. Chick, P. J. Sanchez, D. Ferrin, D. Morrice, eds., Proceedings of the 2003 Winter Simulation Conference, Institute of Electrical and Electronics Engineers, Inc., New Orleans, Lousiana, pp. 13191325, 2003.

[9] F. Zouaoui, J.R. Wilson, B.A. Peters, J.S. Smith, D.J. Medeiros, M.W. Rohrer; "Accounting for input model and parameter uncertainty in simulation", eds., Proceedings of the 2001 Winter Simulation Conference, Institute of Electrical and Electronics Engineers, Inc., Arlington, Virginia, pp. 290-299, 2001 . 